$\mathrm{H}$ еобходимость комплексного подхода к терапии у женщин

\author{
с телогеновым выпадением волос, ассоциированным \\ с железодефицитным состоянием
}

(С Гайдина Т.А. ${ }^{*}$ Щербина С.А., Скрипкина П.А., Силин А.А.

ФГАОУ ВО РНИМУ им. Н.И. Пирогова Минздрава России

117997, Россия, г. Москва, ул. Островитянова, д. 1

Взаимосвязь нарушения обмена железа и алопеции, в частности телогенового выпадения волос (TBB), сохраняется недостаточно изученной и требует дальнейших исследований.

Цель. Оценить изменения динамики роста и состояния волос у женщин с железодефицитным состоянием при системной терапии препаратами железа в комбинации с физиотерапией и без физиотерапии.

Методы. В открытое рандомизированное сравнительное интервенционное исследование длительностью 6 месяцев были включены 26 пациентов женского пола в возрасте от 19 до 54 лет (38,8 \pm 8,9 года) с жалобами на выпадение волос, изменение их структуры, сухость и/или ломкость волос по длине, сохраненным менструальным циклом и железодефицитной анемией (ЖДА) легкой степени тяжести (Нb 101-120 г/л). Проводились трихоскопия и фототрихограмма с обработкой результатов в программе TrichoScienceProv1.1. Оценивалось изменение динамики роста и состояния волос на фроне применения препаратов железа в комбинации с фризиотерапией и без фризиотерапии. В качестве фризиотерапевтического метода лечения ТВВ был выбран импульсный переменный синусоидальный ток высокой частоты, высокого напряжения и малой силы.

Результаты. На фроне проводимой терапии отмечены увеличение содержания $\mathrm{Hb}(p<0,001)$ и положительная динамика трихологических показателей у всех пациентов, выраженность динамики была выше при сочетании терапии препаратами железа с фризиотерапией.

Заключение. Для достижения положительной динамики трихологических показателей у пациентов с телогеновой алопецией на фоне железодефицитного состояния целесообразно использовать комплексное лечение.

Ключевые слова: железодефицитная анемия, латентный дефицит железа, препараты железа, алопеция, телогеновое выпадение волос.

Конфллик интересов: авторы заявляют об отсутствии потенциального конфликта интересов, требующего раскрытия в данной статье.

Для цитирования: Гайдина Т.А., Щербина С.А., Скрипкина П.А., Силин А.А. Необходимость комплексного подхода к терапии у женщин с телогеновым выпадением волос, ассоциированным с железодефицитным состоянием. Вестник дерматологии и венерологии. 2020;96(5):39-46.

doi: https://doi.org/10.25208/vdv539-2020-96-5-39-46 


\title{
1 he necessity of holistic approach to treatment of telogen hair loss associated with iron deficiency in women
}

\author{
(C) Tatiana A. Gaydina*, Sofia A. Shcherbina, Polina A. Skripkina, Alexey A. Silin
}

\author{
Pirogov Russian National Research Medical University \\ Ostrovityanova str., 1, Moscow, 117997, Russia
}

The relationship between disorders of iron metabolism and alopecia, in particular telogen hair loss (THL), remains poorly understood and requires further research.

Goal. To assess changes in the dynamics of growth and hair condition in women with an iron deficiency state during systemic therapy with iron supplements in combination with and without physiotherapy. Methods. An open-label, randomized, comparative interventional study with a duration of 6 months included 26 female aged 19 to 54 years (38.8 \pm 8.9 years) with complaints of hair loss, changes in their structure, dryness and / or hair brittleness; with preserved menstrual cycle and mild iron deficiency anemia (IDA, Hb 101-120 g/l). Trichoscopy and phototrichogram were performed with processing of the results in the TrichoScienceProv1.1 program. The changes in the dynamics of hair growth and condition were assessed against the background of the use of iron supplements in combination with physiotherapy and without physiotherapy. Pulsed alternating sinusoidal current of high frequency, high voltage and low strength was chosen as a physiotherapeutic method for the treatment of THL.

Results. Against the background of the therapy, an increase in the $\mathrm{Hb}$ level $(p<0.001)$ and a positive dynamics of trichological parameters in all patients were noted, the severity of which was higher when the therapy with iron supplements was combined with physiotherapy.

Conclusion. Our findings confirm that the complex treatment of patients with telogen alopecia on the background of an iron deficiency state may achieve positive dynamics of trichological indicators.

Keywords: iron deficiency anemia, latent iron deficiency, iron supplementions, alopecia, telogen hair loss.

Conflict of interest: the authors state that there is no potential conflict of interest requiring disclosure in this article.

For citation: Gaydina TA, Shcherbina SA, Skripkina PA, Silin AA. The necessity of holistic approach to treatment of telogen hair loss associated with iron deficiency in women. Vestnik Dermatologii i Venerologii. 2020;96(5):39-46. doi: https://doi.org/10.25208/vdv539-2020-96-5-39-46 
Сокращения:

ЖДА - железодефицитная анемия

ПЖ - препараты железа

ЛДЖ - латентный десицит железа

ТВВ - телогеновое выпадение волос

АГА - андрогенная алопеция

ВЧГ - волосистая часть головы

$\mathrm{Hb}$ - гемоглобин
Reductions:

IDA - iron deficiency anemia

IS - iron supplementions

LID — latent iron deficiency

$\mathrm{THL}$ - telogen hair loss

$\mathrm{AA}$ - androgenic alopecia

$S$ - scalp

$\mathrm{Hb}$ - hemoglobin

\section{Введение}

За последнее десятилетие количество пациентов с различными формами алопеций значительно возросло [1]. По данным исследователей у пациентов, обратившихся к дерматологу с проблемой выпадения волос, первое место по встречаемости занимает андрогенная алопеция (АГА), которая наблюдается более чем у 50\% мужчин и женщин [2, 3]. Второе место среди всех алопеций занимает телогеновое выпадение волос (ТВB) [4]. Неуклонно растет количество пациентов с сочетанием различных форм потери волос, особенно комбинации АГА с другими фрормами алопеций. Этиологическими факторами дифффузной потери волос в фразу телогена могут быть: недостаток нутриентов [5], гормональный дисбаланс [6], хронические стрессы [7], склонность к метаболическим нарушениям, аутоиммунные процессы [8], длительный прием лекарственных препаратов [9], наличие очагов хронической инфекции [10, 11]. Выявление этиологических факторов имеет ключевое значение в терапии любых форм алопеций. Для постановки диагноза используются общеклинические (сбор анамнеза, осмотр, общий и биохимический анализы крови, анализ крови на гормоны) и трихологические методы исследования (фототрихограмма, pull-тест, микроскопия корня волоса) [12]. Ведущую роль в лечении TBB играет системная терапия, направленная на устранение этиологических фракторов [13, 14]. Применение дополнительных методов лечения (физиотерапии, мезотерапии, терапии аутоплазмой, обогащенной тромбоцитами) позволяет получить значительное клиническое улучшение у пациентов с ТВВ [1, 15-20].

У женщин одним из фракторов, который может негативно влиять на нормальный рост волос и своевременную их цикличную смену, является десиццит железа в организме [21]. ЖДА и латентный десицит железа (ЛДЖ) в большинстве клинических случаев, по мнению экспертов Всемирной организации здравоохранения, развивается у лиц обоих полов вследствие несбалансированного питания [22]. Также ЖДА и ЛДЖ могут развиться вследствие хронических кровопотерь. У женщин детородного возраста причиной железодефицитных состояний могут быть обильные маточные кровотечения [23]. Распространенность железодефицитных состояний, особенно латентно протекающих у женщин молодого и среднего возраста, приобретает медико-социальную значимость, а наличие ТВВ является одним из сигналов специалистам первичного звена для более тщательного обследования и решения вопроса о лечении пациентов.

Современные исследования вносят существенный вклад в понимание взаимосвязи между метаболизмом железа и ростом волос. Единого мнения по этому вопросу на сегодняшний момент не существует, поэтому требуется дальнейшее тщательное изучение взаимосвязи железодефицитного состояния и алопеции, что и явилось основанием для проведения нашего исследования.
На сегодняшний день мнение специалистов относительно влияния ЖДА и ЛДЖ на индукцию или течение ТВВ неоднозначно [24-26]. Часть отечественных и зарубежных специалистов склоняется к мнению, что ТВВ может быть ассоциировано с дефицитом железа [27, 28], однако некоторые исследователи не видят корреляции между этими состояниями и выпадением волос. М. Moeinvaziri с соавт. отмечает, что женщины с железодефицитным статусом находятся в группе риска по ТВВ. Авторы считают, что важную роль в выпадении волос играет сывороточный ферритин, а также существует корреляция между TВВ и уровнем сывороточного фрерритина ниже 30 нг/мл у здоровых женщин [29, 30]. Ряд исследователей считают, что дефицит железа не оказывает влияния на выпадение волос, но при этом не исключают, что при достижении определенного нижнего порога уровня железа в крови (фрерритин ниже 10 мкг/л) возможна индукция уже существующих процессов [31, 32].

Olsen E.A. с соавторами провели обследование женщин в возрасте старше 18 лет, страдающих АГА и/или TBB и не имеющих проблемы выпадения волос в анамнезе. Оценивались уровни ферритина и гемоглобина в сыворотке крови и менопаузальный статус. В результате не было отмечено статистически значимого увеличения частоты десрицита железа у женщин в пременопаузе или постменопаузе с АГА или ТВВ по сравнению с контрольными группами [33]. Xin Du и соавт. провели фундаментальное исследование, в котором сообщили об алопеции на теле железодефицитных мышей в результате мутации белка гена TMPRSS6 матриптазы-2, которая отрицательно регулирует ген Нamp, кодирующий белок гепсидин. Гепсидин подавляет всасывание железа из кишечника и ингибирует его высвобождение из макрофрагов. В рамках исследования применение добавок железа у подопытных мышей не только устранило железодефицит, но и восстановило рост волос [34].

\section{Цель}

Оценить изменения динамики роста и состояния волос у женщин с железодефицитным состоянием при системной терапии препаратами железа в комбинации с физиотерапией и без фризиотерапии.

\section{Материал и методы}

В открытое рандомизированное сравнительное интервенционное исследование продолжительностью 6 месяцев были включены 26 пациентов женского пола в возрасте от 19 до 54 лет (38,8 \pm 8,9 года) с coхраненным менструальным циклом и железодефицитной анемией легкой степени тяжести (уровень гемоглобина менее 120 г/л, но более 100 г/л) вследствие алиментарного генеза. Пациенты обращались к трихологу с жалобами на выпадение волос, изменение структуры волос, сухость и/или ломкость волос по длине. 
Критерием исключения из исследования являлись мужской пол, возраст до 18 и старше 55 лет, беременность и лактация, длительность алопеции менее 6 месяцев, нежелезодефицитные анемии, острые заболевания или обострение хронических, самостоятельный прием во время лечения лекарственных препаратов и биологически активных добавок, содержащих микроэлементы.

Все пациенты подписали добровольное информированное согласие на участие в исследовании.

Всем пациентам проводились трихоскопия и фототрихограмма (аппарат Aramo-SGDiagnosis System). Обработка результатов проводилась в компьютерной программе TrichoScienceProv1.1. Для окрашивания волос и постановки дерматологической метки использовались разрешенная для применения на территории РФ краска для волос и краска для татуажа соответственно. При постановке диагноза первостепенное значение имели данные анамнеза и осмотра, анализ фототрихограммы. Участок для фототрихограммы определялся в точке лобно-теменной зоны, стереотаксические координаты которой определялись на 2 см кпереди и вправо от пересечения биаурикалярной и сагиттальной линий. Оценивались следующие параметры: количество волос

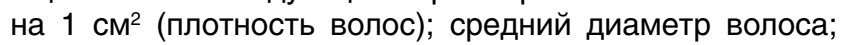
процент веллусных и терминальных волос; процент волос, находящихся в стадии телогена.

Железодефицитное состояние подтверждалось врачом-терапевтом, заключение которого имелось в истории болезни пациента. Анализировались имеющиеся у пациента результаты клинического анализа крови, содержащего информацию об уровне гемоглобина, сывороточного железа и ферритина.

Для коррекции дефицита железа всем пациентам проводилась терапия препаратами железа, терапевтическая доза и длительность приема которых определялись врачом-терапевтом.

Для части пациентов в качестве фризиотерапевтического метода лечения ТВВ был выбран импульсный переменный синусоидальный ток высокой частоты, высокого напряжения и малой силы. Использовался портативный аппарат для дарсонвализации «СпаркСТ-117» ТУ 9444-003-87555850-2012 с принадлежностями. Аппарат допущен к обращению на территории Российской Федерации, выдано регистрационное удостоверение Федеральной службы по надзору в сфрере здравоохранения (РУ ФСР 2012/13527). Протокол процедуры заключался в обработке электродом «гребень» всей поверхности волосистой части головы. В первую процедуру время воздействия составило 7 минут. Со второй процедуры время воздействия постепенно увеличивалось на 1 минуту в каждую последующую процедуру. Максимальное время воздействия составляло 15 минут за одну процедуру. Процедуры проводились 2 раза в неделю в количестве 15 сеансов.

Пациенты методом конвертов были рандомизированы на две группы в зависимости от вида терапии. В первую группу вошли 13 пациентов в возрасте от 19 до $53(36,2 \pm 8,9)$ лет, среди которых у $3(23 \%)$ имелось TВВ и у 10 (77\%) - сочетание АГА и ТВВ. Пациентам первой группы были назначены препараты железа с целью коррекции железодефицитного состояния, а также им проводилось фризиотерапевтическое лечение ТВВ.

Во вторую группу вошли 13 пациентов в возрасте от 32 до $54(42,4 \pm 7,9)$ лет, среди которых преобладали женщины с сочетанием АГА и ТВВ $(n=9,69,2 \%)$ и лишь у 4 (30,8\%) - имелось только ТВВ. Пациенты второй группы получили только препараты железа для коррекции выявленного железодефицитного состояния.

Пациенты посещали дерматолога один раз в месяц в течение полугода. Результаты лечения оценивались через 6 месяцев после начала терапии, с учетом времени, за которое выпадали волосы, перешедшие в телогеновую фразу при дефиците железа, и появились волосы, находящиеся в телогеновой фазе при отсутствии железодефицита у пациентов.

Статистическую обработку данных проводили с использованием пакетов программ SPSS 20.0. Данные представлены в виде $\mathrm{M} \pm \sigma$. Различие считали статистически значимым при значениях двустороннего $p<0,05$.

\section{Результаты}

Среди включенных в исследование пациентов у 7 (27\%) имелось ТВВ и у 19 (73\%) - сочетание АГА и ТВВ; при этом врачом-терапевтом у 10 (38\%) пациентов был установлен диагноз ЖДА, у 16 (62\%) - ЛДЖ. Уровень Нb составил 120,2 \pm 9,8 г/л, сывороточного железа 14,5 \pm 3,7 мкмоль/л, фрерритина - 18,2 \pm 6,2 мкг/л.

Исходно, до лечения, две группы пациентов были сопоставимы по возрасту $(p=0,083)$, уровню $\mathrm{Hb}$ $(p=0,520)$, сывороточного железа $(p=0,069)$ и ферритина $(p=0,582)$. В первой группе пациентов уровень Нb составил $119,1 \pm 11,0$ г/л, концентрация сывороточного железа - 13,4 $\pm 2,9$ мкмоль/л, фрерритина $17,5 \pm 6,6$ мкг/л. Во второй группе пациентов уровень $\mathrm{Hb}$ составил $121,9 \pm 8,1$ г/л, концентрация сывороточного железа - 16,1 \pm 4,1 мкмоль/л, фрерритина $18,9 \pm 5,2$ мкг/л.

На фроне проводимой терапии отмечено увеличение содержания $\mathrm{Hb}$ как у пациентов первой группы до $133,3 \pm 5,4$ г/л $(p<0,001)$, так и у пациентов второй группы - до $131,9 \pm 6,4$ г/л $(p<0,001)$. Концентрация сывороточного железа и ферритина в первой группе пациентов составила 16,9 $\pm 3,4$ мкмоль/л л $(p=0,010)$ и 46,7 $\pm 11,2$ мкг/л л $(p<0,001)$, во второй $-16,2 \pm 2,2$ $(p=0,170)$ мкмоль/л и 46,9 $\pm 11,6$ мкг/л л $(p<0,001)$, соответственно.

Фототрихограммы до и после лечения у пациента из первой и у пациента из второй группы представлены на рис. $1-4$.

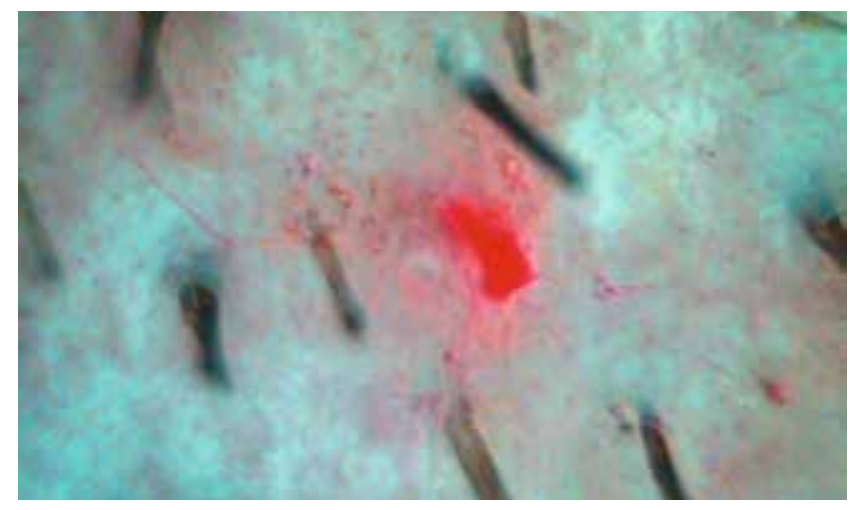

Рис. 1. Фототрихограмма пациента с диагнозом ТВВ из первой группь до лечения

Fig. 1. Phototrichogram of a patient diagnosed with THL from the first group before treatment 


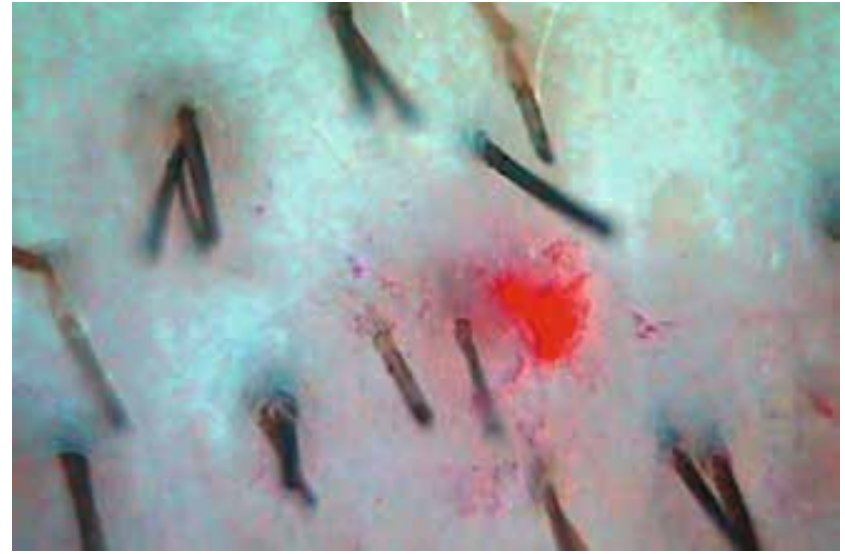

Рис. 2. Фототрихограмма пациента с диагнозом ТВВ из первой группы после лечения

Fig. 2. Phototrichogram of a patient diagnosed with THL from the first group after treatment

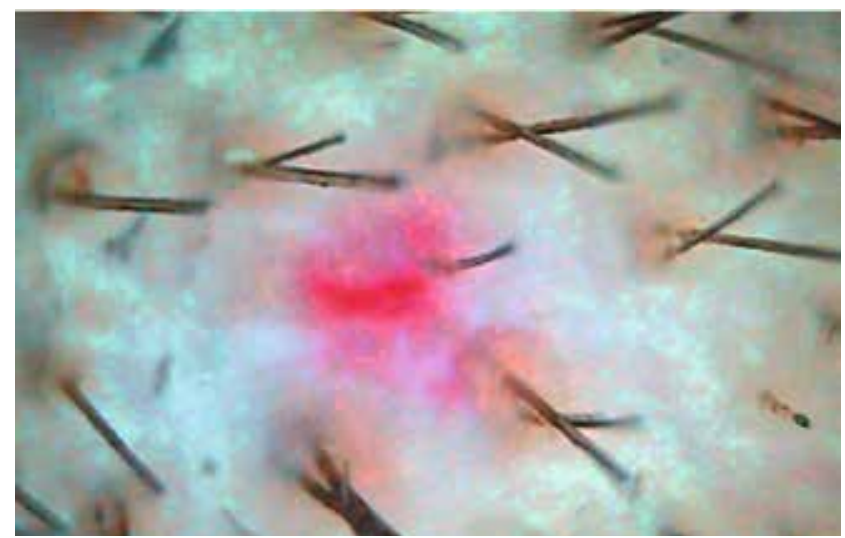

Рис. 4. Фототрихограмма пациента с диагнозом ТВВ + АГА из второй группы после лечения

Fig. 4. Phototrichogram of a patient diagnosed with THL + AA from the second group after treatment

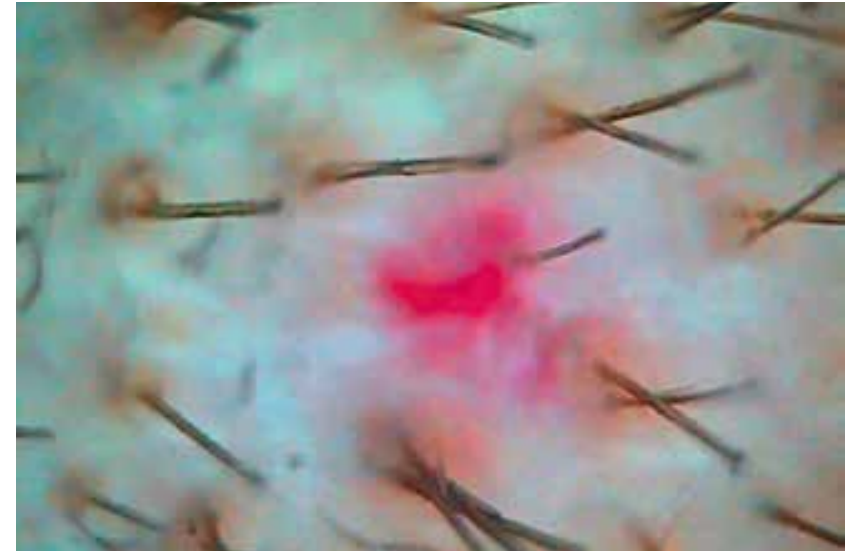

Рис. 3. Фототрихограмма пациента с диагнозом ТВВ + АГА из второй группы до лечения

Fig. 3. Phototrichogram of a patient diagnosed with $T H L+A A$ from the second group before treatment

Распределение средних трихологических показателей в обеих группах до лечения представлено на рис. 5.

На фоне терапии отмечена положительная динамика трихологических показателей в двух группах пациентов, при этом более выраженная динамика наблюдалась у пациентов первой группы по сравнению со второй группой (рис. 6).

Результаты лечения пациентов второй группы представлены на рис. 7.

Таким образом, при сравнении средних трихологических показателей у пациентов обеих групп до и после проведенного лечения было выявлено увеличение общего количества волос на $1 \mathrm{~cm}^{2}$ в лобно-теменной зоне, а также уменьшение количества волос, находящихся в стадии телогена. Такие трихологические показатели, как количество веллусных волос, количество терминальных волос и процент волос в стадии телогена, после лечения у пациентов первой группы имели более выраженную положительную динамику по сравнению

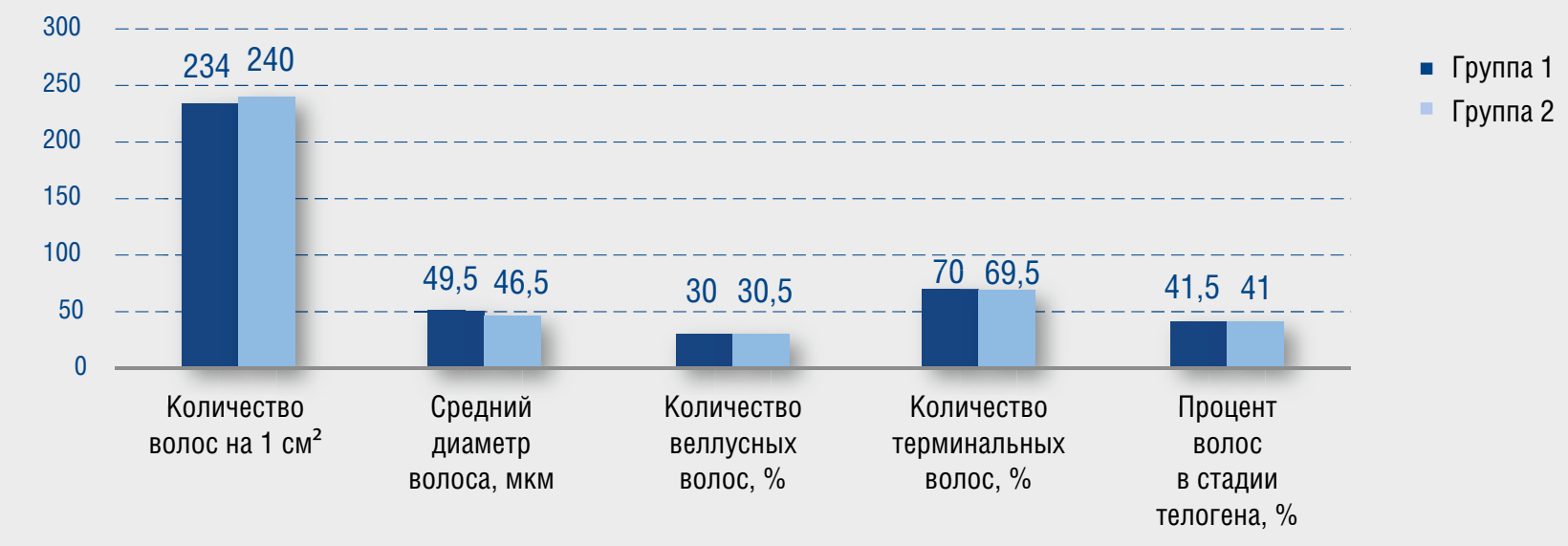

Рис. 5. Средние трихологические показатели пациентов первой и второй групп до лечения

Fig. 5. Average trichological parameters of patients of the first and second groups before treatment 

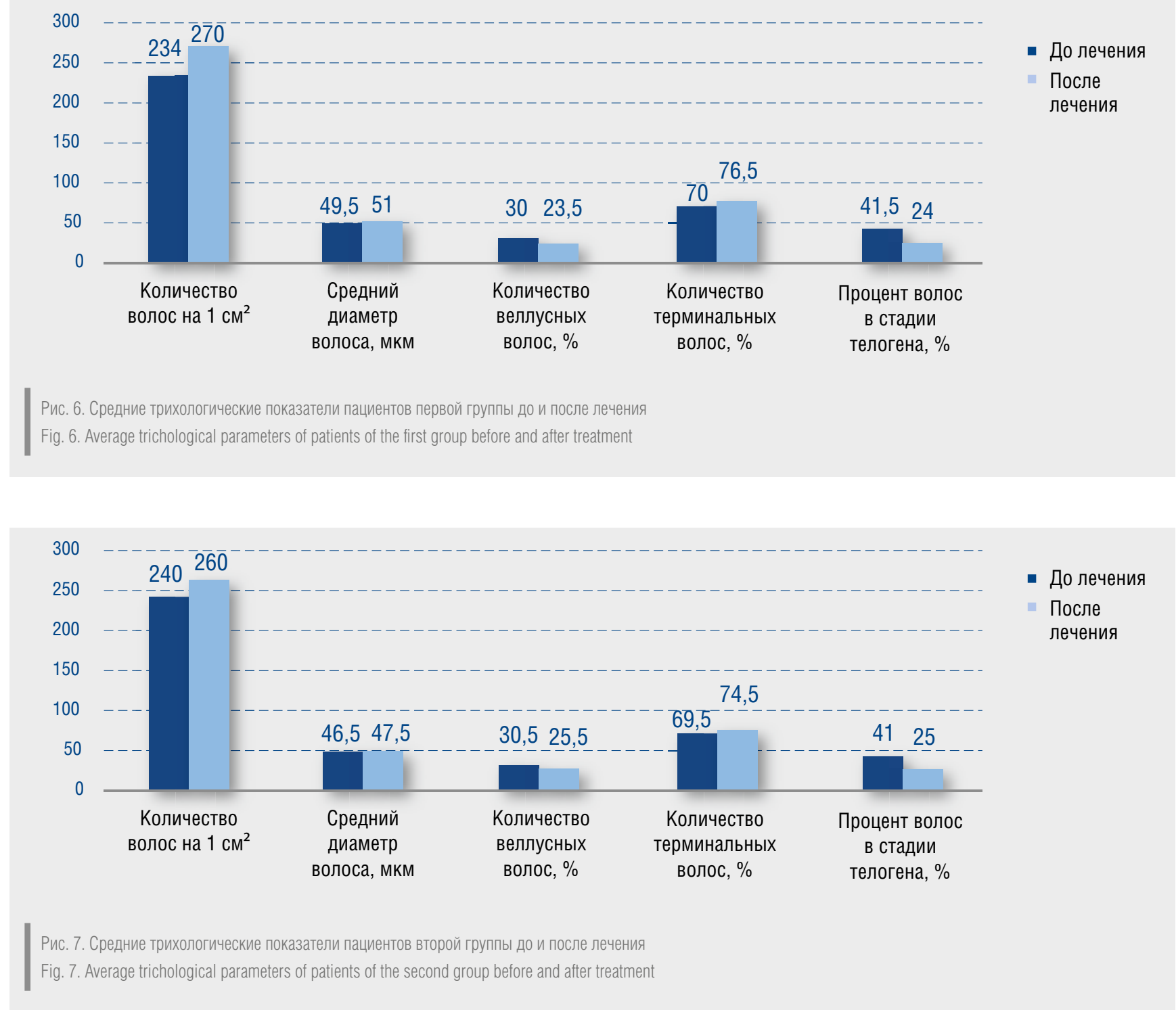

с аналогичными показателями у пациентов второй группы. Тенденция к увеличению диаметра волоса после лечения наблюдалась в обеих группах, но статистически значимых изменений получено не было.

\section{Обсуждение}

Большинство авторов подчеркивают эффрективность комплексного подхода в терапии ТВВ [1, 12], включающего системную терапию и дополнительные методы лечения: фризиотерапию, мезотерапию, терапию аутоплазмой, обогащенной тромбоцитами, что мы и наблюдали на примере наших пациентов. Целью нашей работы была оценка изменения динамики роста и состояния волос у женщин молодого и среднего возраста с сохраненным менструальным циклом и железодесицитным состоянием как при изолированной терапии препаратами железа, так и в комбинации с фризиотерапией. Результаты нашей работы показали улучшение как гематологических, так и трихологических показателей в двух группах пациентов. При этом более выраженный положительный эффрект отмечен у пациентов, которым проводилось комплексное лечение. Особенно следует отметить динамику таких пока- зателей, как уменьшение количества веллусных волос, увеличение количества терминальных волос и снижение процента волос в стадии телогена, что можно объяснить активацией обменных процессов в коже, усилением кровообращения и улучшением трофики тканей в результате дополнительного воздействия на кожу волосистой части головы импульсным переменным синусоидальным током высокой частоты, высокого напряжения и малой силы. Однако мы не отметили значимых изменений диаметра волос в зависимости от лечения, выбранного в группе, что говорит о необходимости подключения дополнительной наружной терапии.

\section{Выводы}

При комплексном лечении препаратами железа и фризиотерапией у женщин с железодефицитным состоянием наблюдалась более выраженная динамика роста волос и улучшения их состояния, чем на фоне лечения только препаратами железа. Полученные результаты позволяют рассматривать системную терапию препаратами железа в комбинации с физиотерапией в качестве возможного варианта лечения у женщин с ТВВ, ассоциированным с железодефицитным состоянием. 


\section{Литература/References}

1. Кубанов А.А., Галлямова Ю.А., Селезнева О.А. Исследование эффрективности комплексной терапии выпадения волос. Вестник дерматологии и венерологии. 2016;(1):32-46. [Kubanov AA, Galljamova JA, Selezneva OA. Efficiency research of the comprehensive hair loss treatment. Vestnik dermatologii i venerologii. 2016;(1):32-46 (In Russ.)]

2. Vano-Galvan S, Saceda-Corralo D, Blume-Peytavi U, Cucchía J, Dlova NC, Gavazzoni Dias MFR, et al. Frequency of the Types of Alopecia at Twenty-Two Specialist Hair Clinics: A Multicenter Study. Skin Appendage Disorders. 2019;5(5):309-315. doi: 10.1159/000496708

3. Ozay 0, Arslantas D, Unsal A, Bulur I. The frequency of alopecia and quality of life in high-school students in rural areas (Sivrihisar, Mahmudiye, Alpu, and Beylikova) of Eskisehir. Northern Clinics of Istanbul. 2019;6(3):226-235. doi: 10.14744/nci.2018.59365

4. Звездина И.В., Исаева С.Г., Ляпон А.О. Новый взгляд на терапию хронической идиопатической диффрузной телогеновой алопеции у женщин. Доктор.pу. 2015;7(108):42-46. [Zvezdina IV, Isaeva SG, Lyapon A0. New Insight into Treating Chronic Idiopathic Diffuse Telogen Effluvium in Women. Doktor.ru. 2015;7(108):42-46 (In Russ.)]

5. Almohanna HM, Ahmed AA, Tsatalis JP, Tosti A. The Role of Vitamins and Minerals in Hair Loss: A Review. Dermatology and therapy. 2019;9(1):51-70. doi: 10.1007/s13555-018-0278-6

6. Vinay K, Sawatkar GU, Dogra S. Hair manifestations of endocrine diseases: A brief review. Indian Journal of Dermatology, Venereology, and Leprology. 2018;84(5):528-538. doi: 10.4103/ijdvl.IJDVL_671_17

7. Phillips TG, Slomiany WP, Allison R. Hair Loss: Common Causes and Treatment. American Family Physician. 2017;96(6):371-378.

8. Rebora A. Telogen effluvium: a comprehensive review. Clinical, cosmetic and investigational dermatology. 2019;(12):583-590.

doi: $10.2147 / C C I D . S 200471$

9. Ghias MH, Amin BD, Kutner AJ. Albendazole-induced anagen effluvium Journal of the American Academy of Dermatology Case Reports. 2020;6(1):54-56. doi: 10.1016/j.jdcr.2019.08.010

10. Chu CB, Yang CC. Dengue-associated telogen effluvium: A report of 14 patients. Dermatologica Sinica. 2017;35(3):124-126.

11. Guzman-Sanchez D, Asz-Sigall D. Alopecias due to drugs and other skin and systemic disorders. Current problems in dermatology. 2015;47:97-106. doi: 10.1159/000369409

12. Кубанов А.А., Галлямова Ю.А., Селезнева О.А. Динамика показателей трихоскопии и фототрихографии у больных нерубцовыми алопециями на фоне комплексного лечения. Лечащий врач. 2016;(5):45. [Kubanov AA, Gallyamova YA, Selezneva OA. Dinamika pokazateley trikhoskopii i fototrikhografii u bolnykh nerubtsovymi alopetsiyami na fone kompleksnogo lecheniya. Lechashchiy vrach. 2016;(5):45 (In Russ.)]

13. Daly T, Daly K. Telogen effluvium with dysesthesia (TED) has lower B12 levels and may respond to B12 supplementation. Journal of drugs in dermatology. 2018:17(11):1236-1240.

14. Karashima T, Tsuruta D, Hamada T, Ono F, Ishii N, Abe T, et al. Oral zinc therapy for zinc deficiency-related telogen effluvium. Dermatologic therapy. 2012;25(2):210-213. doi: 10.1111/j.1529-8019.2012.01443.x

15. Олисова 0.Ю., Егорова К.Г. Богатая тромбоцитами плазма в терапии нерубцовых алопеций. Российский журнал кожных и венерических болезней. 2014;17(6):60-62. [Olisova OY, Egorova KG. Platelet-rich plasma in therapy of nonscarring alopecia. Russian journal of skin and venereal diseases. 2014;17(6):60-62 (In Russ.)]

16. Скадорва В.В. Патогенез, диагностика и лечение дифффузной алопеции. Здравоохранение. 2016;(7):31-38. [Skadorva W. Diffuse alopecia pathogenesis, diagnosis and treatment. Healthcare. 2016;(7):31-38 (In Russ.)]

17. Rangwala S, Rashid RM. Alopecia: a review of laser and light therapies. Dermatology online journal. 2012;18(2):3.
18. Malkud S. Telogen Effluvium: A Review. Journal of clinical and diagnostic research. 2015;9(9):WE01-WE03.

doi: 10.7860/JCDR/2015/15219.6492

19. Stevens J, Khetarpal S. Platelet-rich plasma for androgenetic alopecia: A review of the literature and proposed treatment protocol. International Journal of Women's Dermatology. 2018;5(1):46-51.

doi: 10.1016/j.ijwd.2018.08.004

20. Trüeb RM, Jolliffe VML, Régnier AF, Dutra Rezende H, Vañó-Galván $\mathrm{S}$, Kopera $\mathrm{D}$, et al. Precision Medicine and the Practice of Trichiatry: Adapting the Concept. Skin Appendage Disorders. 2019;5(6):338-343.

doi: $10.1159 / 000500364$

21. Беречикидзе Т.Т., Пинегин В.Б. Диффрузная телогеновая алопеция. Новые возможности коррекции. Российский журнал кожных и венерических болезней. 2016;19(3):162-166. [Berechikidze TT, Pinegin VB. Diffuznaya telogenovaya alopetsiya. Novye vozmozhnosti korrektsii. Rossiyskiy zhurnal kozhnykh i venericheskikh bolezney. 2016;19(3):162166 (In Russ.)]

22. World Health Organization. Haemoglobin concentrations for the diagnosis of anaemia and assessment of severity. Vitamin and Mineral Nutrition Information System. Geneva: WHO; 2011.

23. Camaschella C. Iron deficiency. Blood. 2019;133(1):30-39.

doi: 10.1182/blood-2018-05-815944

24. Gowda D, Premalatha V, Imtiyaz DB. Prevalence of nutritional deficiencies in hair loss among Indian participants: Results of a cross-sectional study. International journal of trichology. 2017;9(3):101-104.

doi: 10.4103/ijt.ijt_48_16

25. Deo K, Sharma Y, Wadhoka, M, Tyagi N. Clinicoepidemiological Observational Study of Acquired Alopecias in Females Correlating with Anemia and Thyroid Function. Dermatology Research and Practice. 2016;(1):1-5.

doi: 10.1155/2016/6279108

26. Grover C, Khurana A. Telogen effluvium. Indian journal of dermatology, venereology and leprology. 2013;79(5):591-603.

doi: 10.4103/0378-6323.116731

27. Perez-Mora N, Goren A, Velasco C, Bermudez F. Acute telogen effluvium onset event is associated with the presence of female androgenetic alopecia: potential therapeutic implications. Dermatologic therapy. 2014;27(3):159-162. doi: 10.1111/dth.12101

28. Trüeb RM. Effect of ultraviolet radiation, smoking and nutrition on hair. Current problems in dermatology. 2015;47:107-120.

doi: $10.1159 / 000369411$

29. Moeinvaziri M, Mansoori P, Holakooee K, Safaee Naraghi Z, Abbasi $A$. Iron status in diffuse telogen hair loss among women. Acta dermatovenerologica Croatica. 2009;17(4):279-284.

30. Poonia K, Thami GP, Bhalla M, Jaiswal S, Sandhu J. NonScarring Diffuse Hair Loss in Women: a Clinico-Etiological Study from tertiary care center in North-West India. Journal of Cosmetic Dermatology. 2019;18(1):401-407. doi: 10.1111/jocd.12559

31. Bregy A, Trüeb RM. No Association between Serum Ferritin Levels $>10 \mu \mathrm{g} / \mathrm{l}$ and Hair Loss Activity in Women. Dermatology. 2008;217(1):1-6. doi: 10.1159/000118505

32. Trüeb R. Telogen Effluvium: Is There a Need for a New Classification? Skin Appendage Disorder. 2016;2:39-44. doi: 10.1159/000446119

33. Olsen EA, Reed KB, Cacchio PB, Caudill L. Iron deficiency in female pattern hair loss, chronic telogen effluvium, and control groups. Journal of the American Academy of Dermatology. 2010;63(6):991-999.

doi: 10.1016/j.jaad.2009.12.006

34. Du X, She E, Gelbart T, Truksa J, Lee P, Xia Y, et al. The serine protease TMPRSS6 is required to sense iron deficiency. Science. 2008;320(5879):1088-1092. doi: 10.1126/science.1157121 


\section{Информация об авторах}

Татьяна Анатольевна Гайдина - К.М.Н., доцент, доцент кафедры дерматовенерологии лечебного фракультета ФГАОУ ВО РНИМУ им. Н.И. Пирогова Минздрава России; тел.: +7 (916) 202-88-90; ORCID ID: 0000-0001-8485-3294; eLibrary SPIN: 5216-2059; e-mail: doc429@yandex.ru

София Андреевна Щербина - ассистент кафедры топограсической анатомии и оперативной хирургии педиатрического фракультета ФГАОУ ВО РНИМУ им. Н.И. Пирогова Минздрава России; ОRCID ID: 0000-0002-2936-0021

Полина Александровна Скрипкина - к.М.Н., доцент, доцент кафедры дерматовенерологии лечебного факультета ФГАОУ ВО РНИМУ им. Н.И. Пирогова Минздрава России; ORCID ID: 0000-0001-9953-1095; eLibrary SPIN: 3706-1349

Алексей Александрович Силин - инспектор деканата лечебного фракультета ФГАОУ ВО РНИМУ им. Н.И. Пирогова Минздрава России; ORCID ID: 0000-0003-0312-4853

\section{Information about the authors}

Tatiana A. Gaydina - Candidate of Medical Sciences, Associate Professor, Associate Professor at the Department of dermatovenerology medical faculty of Pirogov Russian National Research Medical University; tel.: +7 (916) 202-88-90; ORCID ID: 0000-0001-8485-3294; eLibrary SPIN: 5216-2059; e-mail: doc429@yandex.ru

Sofia A. Shcherbina - Assistant at the Department of topographic anatomy and operative surgery of the pediatric faculty of Pirogov Russian National Research Medical University; ORCID ID: 0000-0002-2936-0021

Polina A. Skripkina - Candidate of Medical Sciences, Associate Professor, Associate Professor at the Department of dermatovenerology medical faculty of Pirogov Russian National Research Medical University; ORCID ID: 0000-0001-9953-1095; eLibrary SPIN: 3706-1349

Alexey A. Silin - inspector of the Dean's Office of the Medical Faculty of Pirogov Russian National Research Medical University; ORCID ID: 0000-0003-0312-4853

Статья поступила в редакцию: 14.02 .2020

Принята к публикации: 26.11.2020

Дата публикации: 02.12.2020
Submitted: 14.02 .2020

Accepted: 26.11 .2020

Published: 02.12 .2020 